\title{
Minimally Invasive Decompression Alone for Lumbar Spinal Stenosis with Degenerative Spondylolisthesis: A Prospective Study
}

ISSN: 2576-8875

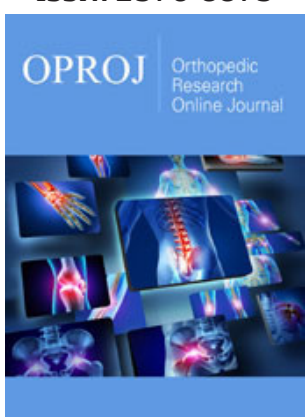

*Corresponding author: Akira Miyauchi, Saka-midorii Hospital, 6-28-1 Midorii, Asaminami-ku, Hiroshima, 7310103, Japan

Submission: 漹 September 25, 2021

Published: 漈October 06, 2021

Volume 9 - Issue 1

How to cite this article: Akira Miyauchi*, Shin-ichi Saka, Hiroshi Terayama. Minimally Invasive Decompression Alone for Lumbar Spinal Stenosis with Degenerative Spondylolisthesis: A Prospective Study. Ortho Res Online J. 9(1). OPROJ. 000702. 2021.

DOI: 10.31031/OPROJ.2021.09.000702

Copyright@: Akira Miyauchi, This article is distributed under the terms of the Creative Commons Attribution 4.0 International License, which permits unrestricted use and redistribution provided that the original author and source are credited.

\author{
Akira Miyauchi' ${ }^{1 *}$, Shin-ichi Saka ${ }^{1}$, Hiroshi Terayama ${ }^{1}$, Yoshifumi Fuse ${ }^{1}$, Kobun \\ Takazawa ${ }^{1}$, Nobukazu Okimoto ${ }^{1}$ and Nobuo Adachi ${ }^{2}$ \\ ${ }^{1}$ Department of Orthopaedic Surgery, Saka-midorii Hospital, Hiroshima, Japan \\ ${ }^{2}$ Department of Orthopaedic Surgery, Hiroshima University Hospital, Japan
}

\begin{abstract}
Background: Minimally invasive decompression has provided satisfactory outcomes with limited slip progression for Lumbar Spinal Stenosis (LSS) with Degenerative Spondylolisthesis (DS). However, how slip progression stops, its impact on clinical symptoms, and whether the patient's sex affects these postdecompressive conditions remain unknown. This study aimed to answer these questions and investigate the effectiveness and limitations of decompression alone for LSS with DS.
\end{abstract}

Methods: We carried out a prospective study which enrolled 99 patients who had LSS with DS at L4/5. Of these, 10 dropped out and 6 required reoperation, leaving 83 patients who underwent microscopic decompression. A 2-year follow-up was carried out. The Japanese Orthopedic Association (JOA) score and visual analog scale for back pain and leg symptoms were used for clinical evaluation. The slip rate (\% slip) in the neutral, flexion, and extension positions; the absolute difference in the $\%$ slip between flexion and extension; and the range of motion were used for radiographic evaluation. A repeated-measures twofactor ANOVA was carried out to determine the differences in each parameter between the sexes and across three different time points (before, and one and two years after surgery).

Results: The remaining 83 patients showed no interactions between sex and changes in parameters at any time point. The slip progression in each position stopped one year after decompression, with the $\%$ slip at the neutral position decreasing 1-2 years after decompression. Mobility did not change, and clinical symptoms improved each year.

Conclusion: The sex of the patient did not affect the post-decompressive course, and slip progression stopped one year after decompression, with no change in mobility. This post-decompressive restabilization can improve clinical symptoms 1-2 years after decompression.

Keywords: Minimally invasive decompression; Lumbar spinal stenosis; Degenerative spondylolisthesis

\section{Introduction}

Lumbar Degenerative Spondylolisthesis (DS) is a self-limiting disorder; spondylosis deformans of the lumbar spine can ultimately halt slip progression and lead to restabilization [1-3]. Minimally Invasive Decompression (MID) can provide leverage in the surgical treatment of Lumbar Spinal Stenosis (LSS) with DS. When the damage to the posterior supporting elements is minimal, post-decompressive restabilization can be attained with limited slip progression at some point after MID. Thus, the neural decompression effect can be maintained without spinal fusion. In fact, MID alone has consistently provided satisfactory surgical outcomes [4-8]. However, simple decompression is characterized by degenerative changes, such as increased slipping and disc herniation, when compared with spinal fusion. These changes account for most negative surgical outcomes and revision surgeries [9-12]. Additionally, DS features a female prevalence in the progression and grade of slipping $[3,13]$. Considering these, the following issues concerning the effectiveness and limitations of decompression alone for LSS with DS must be clarified: (1) how and when post-decompressive slip progression stops, allowing restabilization; (2) how radiographic changes affect 
postoperative clinical symptoms; and (3) the potential existence of sex-related differences influencing the answers to those questions.

This study aimed to address the above in a prospective manner.

\section{Materials and Methods}

This prospective study was initiated in April 2014. Notably, during follow-up, the slip progression stopped in many patients, approximately one year after decompression [8]. Therefore, we conducted a 2-year follow-up and analyzed the data. The radiographic findings and clinical symptoms were evaluated annually according to the parameters described below.

All patients were informed of the surgery and the purpose of this study, and they all provided written informed consent before surgery. The inclusion criteria were: 1) the presence of LSS with a slip rate (\% slip) $\geq 10 \%$ at L4-L5 in the neutral position on a standing radiograph, 2) radiculopathy or neurogenic claudication disturbing daily activities, with associated neurological signs consistent with LSS at L4-L5, and 3) microscopic decompression performed at L4-L5 without spinal fusion. The exclusion criteria were: 1) any previous lumbar surgery, 2) concomitant disc herniation, or 3) radiculopathy due to intra- and/or extra-foraminal stenosis. Female sex hormones are related to the progression and grade of DS [1416]. Thus, we also excluded female patients receiving hormone replacement therapy for postmenopausal conditions and/or who had an oophorectomy prior to surgery or during the followup period. Surgery was performed by the first author and our mentor who passed away during the study period, both of whom have more than 20 years of experience in performing microscopic decompression

Nighty-nine patients were enrolled between April 2014 and March 2019. Ten patients dropped out: one patient developed senile dementia; one patient died (cancer); two patients developed Parkinson's disease; and six patients declined to participate for $>1$ year of follow-up because of improved symptoms. The remaining 89 patients were evaluated in this study. There were 26 males and 63 females, with an average age of $69.0 \pm 9.3$ years during surgery (mean \pm standard deviation).

\section{Clinical evaluation}

Clinical outcomes were determined using the Japanese Orthopedic Association (JOA) 15-point scoring system for assessing function (0-15 points, where 0 indicated the greatest severity; Table 1), a Visual Analog Scale (VAS) for lower back pain (B-VAS: $0-10$, where 10 indicated the most severe pain) and leg symptoms (L-VAS: 0-10 for pain and 0-10 for numbness; a score of 20 indicated the greatest symptom severity).

Table 1: Scoring system for low back pain proposed by the Japanese Orthopedic Association (maximum 15 points).

\begin{tabular}{|c|}
\hline Subjective Symptoms (9 Points) \\
\hline Low back pain \\
\hline $3=$ None \\
\hline $2=$ Occasional mild pain \\
\hline $1=$ Frequent mild or occasional severe pain $0=$ Frequent or continuous severe pain \\
\hline Leg pain and/or tingling \\
\hline $3=$ None \\
\hline $2=$ Occasional slight symptoms \\
\hline $1=$ Frequent mild or occasional severe symptoms \\
\hline $0=$ Frequent or continuous severe symptoms \\
\hline Gait \\
\hline $3=$ Normal \\
\hline $2=$ Able to walk farther than $500 \mathrm{~m}$, although resulting pain, tingling, and/or muscle weakness \\
\hline 1 = Unable to walk farther than $500 \mathrm{~m}$ due to leg pain, tingling, and/or muscle weakness \\
\hline $0=$ Unable to walk farther than $100 \mathrm{~m}$ due to leg pain, tingling, and/or muscle weakness \\
\hline Clinical Signs (6 Points) \\
\hline Straight leg raise test \\
\hline (Including tight hamstrings) \\
\hline $2=$ Normal \\
\hline $1=30-70^{\circ}$ \\
\hline $0=<30^{\circ}$ \\
\hline Sensory disturbance \\
\hline $2=$ None \\
\hline $1=$ Slight disturbance (not subjective) \\
\hline $0=$ Marked disturbance \\
\hline Motor disturbance (MMT) \\
\hline $2=$ Normal (grade 5) \\
\hline 1 = Slight weakness (grade 4) \\
\hline $0=$ Marked weakness (grade 3-0) \\
\hline
\end{tabular}

MMT: Manual Muscle Test 


\section{Radiographic evaluation}

The \% slip was calculated through standing lateral radiographs in three positions (i.e., flexion, neutral, and extension). Translational and angular mobility at L4-L5 were evaluated based on the absolute difference between the $\% \operatorname{slip}(\Delta \%$ slip) measured in the flexion and extension positions and the Range of Motion (ROM) from extension to flexion. Finally, although the change in the disc height is a valuable parameter for evaluating degeneration of the intervertebral disc after decompression, it was difficult to set standard measuring points due to the various morphologies within the disc space that occur with DS during follow-up (Figure 1a \& 1f; [8]). Therefore, we excluded the evaluation of disc height from this study.

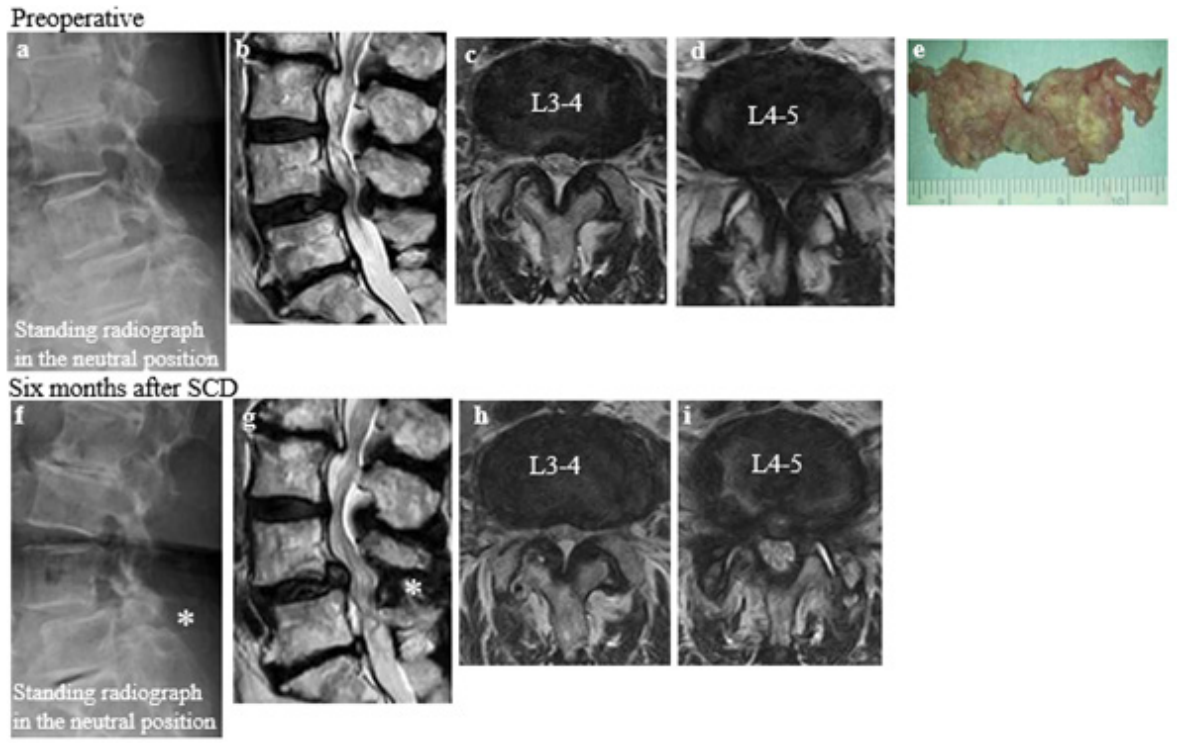

Figure 1: (cited from [8]) A case requiring reoperation after Semi-Circumferential Decompression (SCD, a midsagittal to bilateral microscopic decompression) at L4-5 (Table 3, Case 6). The hypertrophied ligamentum flavum at L4-5 (b, d) had been completely removed (e) with maximal preservation of the facet joints (i) and spinous processes ( $\mathrm{f}, \mathrm{g}$ asterisk $\leq 10 \mathrm{~mm}$ between the processes). Compensating flexion as a pain-relieving posture (a) was improved after SCD with developed lumbar lordosis (f), which caused the ligamentum flavum hypertrophy and symptomatic LSS at L3-4 (b, c, g, h).

\section{Statistical analysis}

Statistical analyses were performed using Bell Curve for Excel (Social Survey Research Information Co., Ltd.). We performed a repeated-measures two-factor analysis of variance (ANOVA) to determine the difference in each clinical and radiographic parameter evaluated between the patients' sex and across three different time points (i.e., before, and one and two years after surgery), and the interaction between sex and the changes in each parameter across these time points. Mauchly's sphericity test was performed to validate the ANOVA. In cases where $\mathrm{P}<0.05$, Greenhouse-Geisser correction was used; otherwise, a conservative F-test was used. For post-hoc testing, the Wilcoxon signed-rank test was performed with a Bonferroni correction. For the other statistical analyses, the Mann-Whitney U and chi-squared independence tests were used. A risk of $5 \%$ was considered statistically significant.

\section{Surgical method}

The microscopic decompression method used, semicircumferential decompression (SCD), features en-bloc removal of the degenerative ligamentum flavum and involves minimal damage to the facet joints and the spinal processes $[5,8,17]$. After partial resection of the spinal processes of $\mathrm{L} 4$ and $\mathrm{L} 5$, holding $\leq 10 \mathrm{~mm}$ between the two processes, decompression was performed midsagittal to bilateral. Bilateral nerve roots were decompressed from bony entrapment. In addition, neural compression exerted by the epidural membrane and periradicular fibrous tissues were relieved by releasing these tissues from the dura [17]; (Figure 1).

\section{Results}

Table 2 shows the evaluated patients' characteristics. The preoperative JOA, B-VAS, and L-VAS scores were $9.0 \pm 1.7,4.7 \pm 3.1$ and $14.9 \pm 3.8$, respectively. The preoperative $\%$ slip was $19.2 \% \pm 5.6 \%$ at flexion, $17.1 \% \pm 5.2 \%$ at neutral position, and $15.5 \% \pm 5.6 \%$ at extension. For mobility, the $\Delta \%$ slip was $3.8 \% \pm 2.7 \%$ and the ROM was $6.3^{\circ} \pm 3.8^{\circ}$.

The male and female patients were similar in terms of age at surgery, duration of symptoms, and preoperative JOA, B-VAS, and L-VAS scores. Females displayed a greater $\%$ slip in each position than males, but the $\Delta \%$ slip and ROM did not differ between them.

There were no dural tears, and reoperations were not required to manage postoperative epidural hematomas or infections.

\section{Reoperation cases (Table 3)}

During follow-up, six patients (6.7\%; one male and five females) required reoperations. Four patients suffered radiculopathy due to 
degeneration at the decompressed L4-L5 level: foraminal stenosis (one patient), intraforaminal disc herniation (one patient), and juxta facet cyst (two patients). The remaining two patients suffered leg symptoms due to stenosis at L3-L4 (Figure 1; [8]). The reoperation rate did not differ between males and females (Table $4, \mathrm{P}=0.43$ ).

Table 2: Preoperative patient characteristics.

\begin{tabular}{|c|c|c|c|c|}
\hline & All Patients ( $n=89)$ & Male $(n=26)$ & Female $(n=63)$ & $\mathbf{P}^{*}$ \\
\hline Age at surgery (years) & $69.0 \pm 9.3(45-86)$ & $71.3 \pm 8.9(51-86)$ & $68.1 \pm 9.3(4-86)$ & 0.31 \\
\hline $\begin{array}{l}\text { Symptom duration } \\
\text { (months) }\end{array}$ & $32.2 \pm 39.7(1.2-207.8)$ & $32.2 \pm 33.9(2.9-122.1)$ & $32.2 \pm 42.2(1.2-207.8)$ & 0.67 \\
\hline Median & 17.5 & 18 & 16.9 & \\
\hline \multicolumn{5}{|l|}{ Clinical evaluation } \\
\hline JOA score & $9.0 \pm 1.7(4-12)$ & $9.3 \pm 1.8(6-12)$ & $8.9 \pm 1.6(4-12)$ & 0.22 \\
\hline B-VAS & $4.9 \pm 3.0(0-10)$ & $4.8 \pm 3.3(0-10)$ & $4.9 \pm 3.0(0-10)$ & 0.86 \\
\hline L-VAS & $14.9 \pm 3.8(3.2-20)$ & $14.6 \pm 3.0(7.1-20)$ & $15.0 \pm 4.0(3.2-20)$ & 0.42 \\
\hline \multicolumn{5}{|l|}{ Radiographic evaluation } \\
\hline \multicolumn{5}{|l|}{ \% Slip (\%) } \\
\hline Neutral position & $17.1 \pm 5.2(10.0-29.1)$ & $13.0 \pm 3.4(10.0-24.7)$ & $18.7 \pm 4.9(10.9-29.1)$ & $<0.01$ \\
\hline Flexion & $19.2 \pm 5.6(10.3-30.7)$ & $14.2 \pm 3.4(10.3-25)$ & $21.3 \pm 5.0(12.0-30.7)$ & $<0.01$ \\
\hline Extension & $15.5 \pm 5.6(6.9-28.7)$ & $11.0 \pm 3.5(6.9-24)$ & $17.3 \pm 5.3(7.4-28.7)$ & $<0.01$ \\
\hline$\Delta \%$ slip (\%) & $3.8 \pm 2.7(0.0-13.1)$ & $3.4 \pm 2.0(0.0-6.7)$ & $4.0 \pm 2.9(0.3-13.1)$ & 0.63 \\
\hline $\mathrm{ROM}\left({ }^{\circ}\right)$ & $6.3 \pm 3.8(0.0-15.0)$ & $6.8 \pm 3.6(2.0-13.8)$ & $6.1 \pm 3.9(0.0-15.0)$ & 0.36 \\
\hline
\end{tabular}

Values are shown as mean \pm SD (range).

*Male vs. female; Mann-Whitney U test.

\section{Patients not requiring reoperation}

From the remaining 83 patients (25 males and 58 females), radiographic and clinical parameters were evaluated throughout the 2-year follow-up.

Changes in clinical symptoms throughout the followup (Figure 2): Mauchly's sphericity test revealed statistical significance in the JOA, B-VAS, and L-VAS scores; thus, GreenhouseGeisser correction was performed for all parameters. Significant differences between male and female patients were not observed for any parameters except the JOA score. The JOA score did not differ between males and females before SCD; however, it was higher in males than in females thereafter. There was no interaction between the patients' sex and changes in each parameter. Significant differences were noted across the three different time points (i.e., before, and 1 and 2 years after SCD) in any parameters: the JOA score increased, and the B- and L-VAS scores decreased each year.

Changes in radiographic findings throughout the follow-up (Figure 3): Mauchly's sphericity test showed statistical significance in the \% slip at each position and ROM, but non-significance in the $\Delta \%$ slip; thus, Greenhouse-Geisser correction was performed for parameters excluding the $\Delta \%$ slip.

The \% slip in each position demonstrated a significant difference between males and females, and across the three different time points. Before and after SCD, females had a higher $\%$ slip in each position than males. However, there was no interaction between the patients' sex and changes in the \% slip in each position across the three different time points. The \% slip in each position increased during the first year after SCD $(\mathrm{P}<0.01)$, but did not change thereafter in flexion and extension. Interestingly, the $\%$ slip in the neutral position decreased between the 1- and 2-year followups after SCD $(\mathrm{P}<0.05)$, which led to the slipping in the neutral position to the same degree as preoperative slipping despite an overall \% slip increase in flexion and extension over the 2 years of follow up.

Despite the changes in the $\%$ slip at each position, the $\Delta \%$ slip and $\mathrm{OM}$ did not differ between males and females, or across the three different time points, and there was no interaction between sex and the changes over time.

\section{Discussion}

DS is a self-limiting disorder with auto stabilization ability [13]. This ability is responsible for the effectiveness of MID in the treatment of LSS with DS. Minimizing damage to the posterior supporting elements, MID alone can lead to satisfactory surgical outcomes with limited slip progression and instability [4-8]. However, how and when the slip progression stops, its impact on clinical symptoms, and whether the female preponderance in DS affects the post-decompressive slip progression and clinical symptoms, remain unknown. These uncertainties cloud the effectiveness of decompression alone for LSS with DS compared with spinal fusion [9-11]. In this context, our study revealed that microscopic decompression resulted in unique postoperative changes in clinical symptoms at the operative DS level, demonstrating the effectiveness and limitations of decompression alone for LSS with DS. 
(a) JOA score
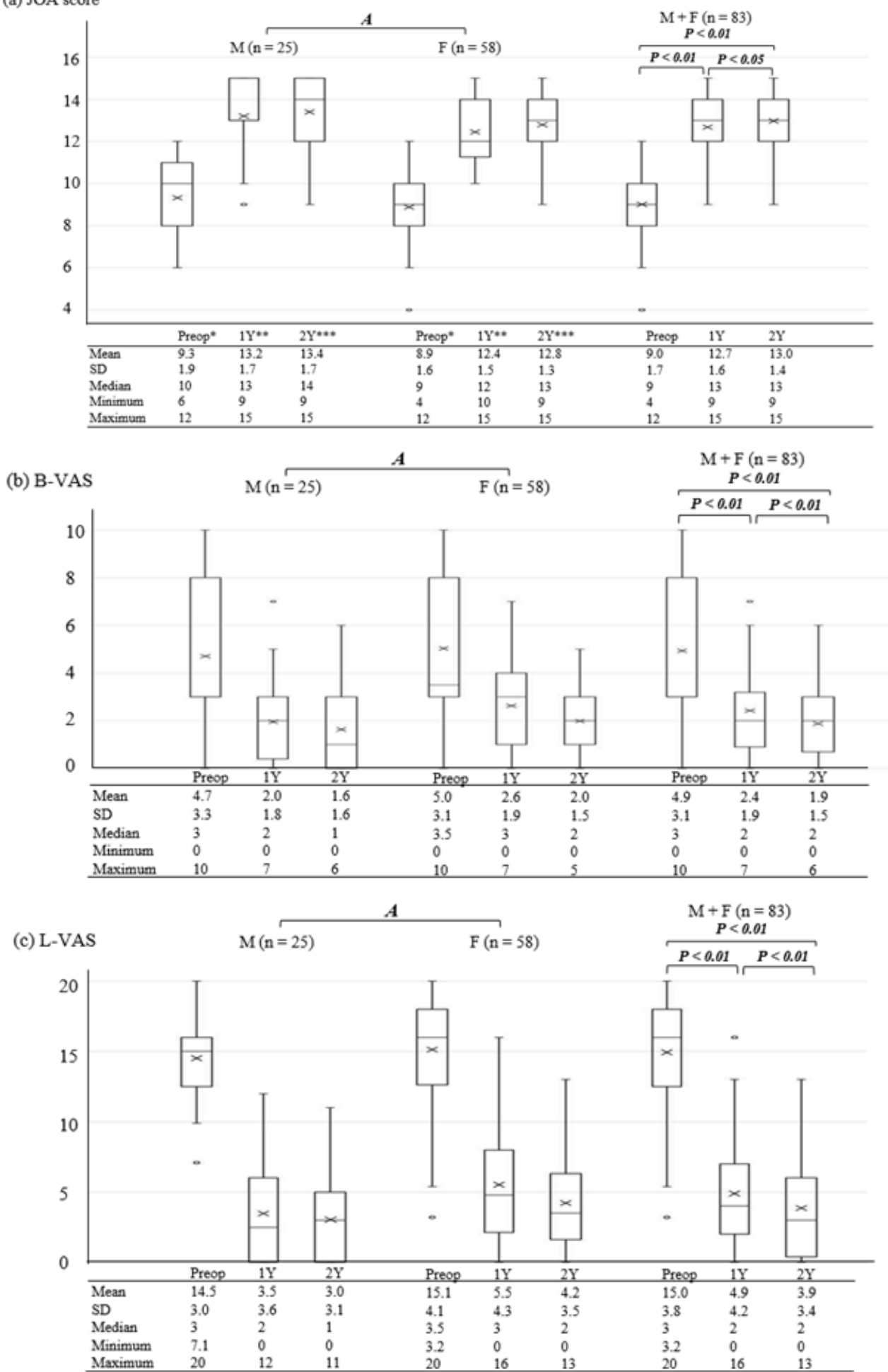

Figure 2: Relationships between sex, time (before, and one and two years after surgery) and three measures of clinical symptoms in patients (a the JOA score, b B-VAS, and c L-VAS). Abbreviations: M, male; F, female; n.s., not significant; Preop, preoperative; $1 \mathrm{Y}$, one-year follow-up; 2Y, two-year follow-up; JOA, Japanese Orthopedic Association; B-VAS, low back pain visual analog scale; L-VAS, leg symptoms visual analog scale. In boxplots, horizontal lines or Me represent the median; $\times$ represents the mean; upper and lower borders of the boxes represent the interquartile range, and circles indicate outliers. Comparison tested via ANOVA (A): a - c Mauchly's sphericity test, $\mathrm{P}<0.01$; Greenhouse-Geisser correction, a $\varepsilon=0.72$, be $=0.65$, and $c \varepsilon=0.67$. Difference between male and female patients: a $\mathrm{P}=0.046$ (Mann-Whitney U-test: ${ }^{*} \mathrm{P}=0.23,{ }^{* *} \mathrm{P}=0.03$, ${ }^{* * *} \mathrm{P}=0.03$ ), b $\mathrm{P}=0.29$, c $\mathrm{P}=0.09$. No interaction between sex and changes across the three timepoints: a $\mathrm{P}=0.65, \mathrm{~b} P=0.71, \mathrm{c} P=0.25$. Significant difference across the three timepoints: a-c $\mathrm{P}<0.01$; for post-hoc testing, Wilcoxon singed-rank test with Bonferroni correction. 
There were no interactions between sex and changes in clinical symptom parameters (JOA, B-VAS, and L-VAS scores) or radiographic findings (the $\%$ slip, $\Delta \%$ slip, and ROM) before, and one and two years after decompression. This result indicates that, despite female prevalence in the progression and grade of slipping, the patients' sex does not affect the post-decompressive course. More specifically, there was no difference between males and females in the mechanisms of clinical symptom improvements, and changes in the slip and mobility at the decompressed segment.

It should be noted that the post-decompressive slip progression was inevitable at each position; however, it stopped one year after decompression. Furthermore, slipping in the neutral position decreased one to two years after decompression, offsetting slip progression in the neutral position during the second year after decompression. Despite the post-operative changes for slipping,

Table 3: Cases requiring reoperation $(n=6,6.7 \%)$. the $\Delta \%$ slip and ROM did not differ before and after decompression. These results demonstrate that MID shifts the center of mobility anteriorly, holding both translational and angular mobility to the same degree. Furthermore, the post-decompressive slip progression should not be identified as increased or having secondary instability. In addition, restabilization can be achieved following the termination of slip progression one year after SCD.

Neural decompression facilitates back extension and the maintenance of a good posture during postoperative daily activities. This postural improvement could have contributed to improving slip progression in the neutral position and relieving lower back pain due to the reduction of the continuous tension in the back muscles and ligaments, and the mechanical load that presses against the intervertebral disc during daily activities $[18,19]$.

\begin{tabular}{|c|c|c|c|c|c|c|}
\hline \multirow{2}{*}{$\begin{array}{l}\text { Case } \\
\text { no. }\end{array}$} & \multirow[b]{2}{*}{ Age, sex } & \multicolumn{3}{|c|}{ Onset of Symptom Recurrence } & \multicolumn{2}{|c|}{ Reoperation } \\
\hline & & $\begin{array}{l}\text { Time after SCD } \\
\text { (months) }\end{array}$ & Symptom & Cause & $\begin{array}{l}\text { Time after SCD } \\
\text { (months) }\end{array}$ & Method \\
\hline 1 & $76, \mathrm{~F}$ & 17 & Right leg pain & Foraminal stenosis at right $\mathrm{L} 4 / 5$ & 24 & Foraminotomy \\
\hline 2 & $73, \mathrm{~F}$ & 12 & Left leg pain & Intraforaminal disc herniation at left $\mathrm{L} 4 / 5$ & 21 & Discectomy \\
\hline 3 & $80, \mathrm{M}$ & 18 & Left leg pain & Facet cyst at left L4/5 & 24 & Cyst resection \\
\hline 4 & $58, \mathrm{~F}$ & 3 & Left leg pain & Facet cyst at left $\mathrm{L} 4 / 5$ & 4 & Cyst resection \\
\hline 5 & $60, \mathrm{~F}$ & 12 & Bilateral leg pain & Stenosis at $\mathrm{L} 3 / 4$ & 13 & SCD \\
\hline 6 & $69, \mathrm{~F}$ & 6 & Bilateral leg pain & Stenosis at $\mathrm{L} 3 / 4$ & 12 & SCD \\
\hline
\end{tabular}

F: female; M: male; SCD: Semi-Circumferential Decompression.

Compared to the B- and L-VAS scores, the post-operative JOA score was higher in male patients, which might be because this scoring system includes the evaluation of gait ability and objective findings. As a result, termination of slip progression, followed by restabilization and postural improvement, can maintain the decompression effect and continually improve clinical symptoms to almost the same degree in male and female patients throughout the follow-up. This is the distinctive postoperative course provided by SCD or MID for LSS with DS. However, such unique postoperative changes indicate the intrinsic drawbacks of decompression alone compared with spinal fusion; this is apparent through the causes of reoperation (Figure 1; [8]; Table 3).

The time required for post-decompressive restabilization is a critical period, especially approximately one year after decompression (at the time of the termination of slip progression). During this period, degenerative changes at the DS segment can accelerate to cause foraminal stenosis, facet joint cysts, or disc herniation along with slip progression. Therefore, surgical outcomes may temporarily deteriorate, and, in some cases, salvage surgery could be required relatively early (i.e., 1-2 years after decompression) $[8,9,11,12,20]$. The addition of spinal fusion may avoid such problems and improve clinical symptoms earlier, due to the forced immediate immobilization, compared with decompression alone. However, the degeneration-related problems in the adjacent segment remain [11,21-24]. These problems are less likely to occur after decompression alone, due to the preservation of mobility at the operated segment $[6,8,11]$.
In addressing the intrinsic drawbacks of MID, described above, minimizing damage to the spinal processes and facet joints is key $[5,7,8,23]$. Adjacent spinal processes, especially in the case of kissing spines, play an important role as a segmental stabilizer, by reducing motion, intradiscal pressure, and the load placed on the facet joints, especially in extension. Impairment of spinous processes cannot be avoided when using a midsagittal to bilateral decompression, as in SCD. This increases the likelihood of postoperative degeneration of the decompressed segment, leading to problems such as disc herniation and facet joint cysts. In contrast, bilateral decompression using a unilateral approach can preserve the spinal processes, but the hypertrophied facet joints and trefoil shaped spinal canal disturb successful and adequate decompression [25]. The disadvantage of each of these approaches must be overcome to increase the surgical reliability of the MID method for LSS with DS.

Decompression alone is associated with fewer complications, lower costs of initial surgery, and a higher revision rate during the relatively early post-operative period compared with spinal fusion $[6,9,20,26]$. Thus, decompression alone and spinal fusion may have comparable cost-utility profiles [20]. Therefore, considering that the definition of symptomatic instability and the indication of spinal fusion have not been established, MID can be the first surgical option for the treatment of LSS with DS. However, the effectiveness and inherent limitations of decompression alone, derived from the unique postoperative course, should be well conveyed to patients and well understood by surgeons. 


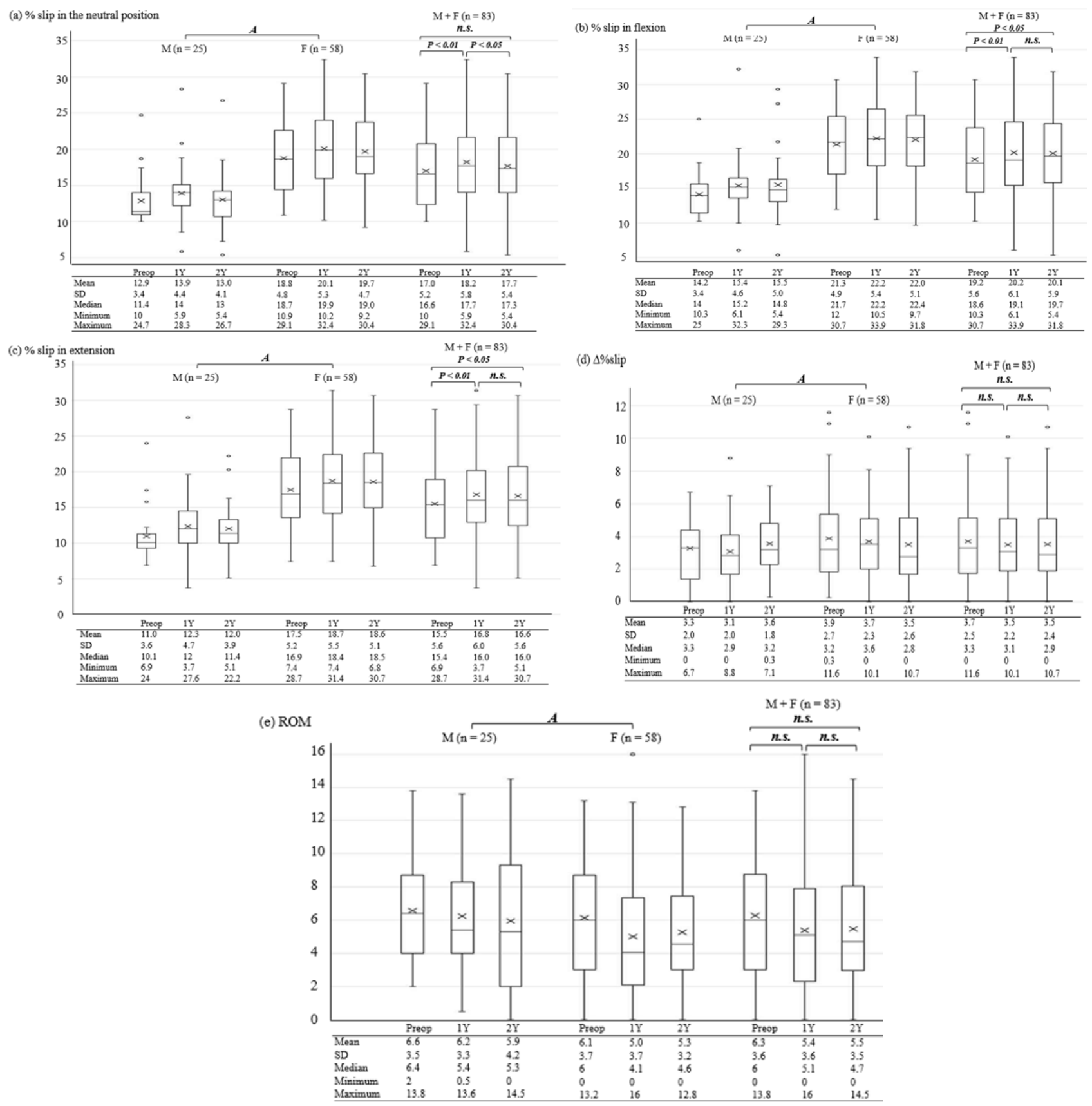

Figure 3: Relationships between sex, time (before, and one and two years after surgery), and radiographic findings in patients (a the \% slip in the neutral position (\%), b the \% slip in flexion (\%), c the \% slip in extension (\%), $\mathrm{d}$ the $\Delta$ $\%$ slip (\%), and e ROM ( $\left.{ }^{\circ}\right)$ ). Abbreviations: Refer to Figure 2. In boxplots, horizontal lines or Me represent the median; $\times$ represents the mean; upper and lower borders of the boxes represent the interquartile range, and circles indicate outliers. Comparisons tested via ANOVA (A): Mauchly's sphericity test, a $\mathrm{P}=0.017$, Greenhouse-Geisser correction, $\varepsilon=0.90 ; \mathrm{b} P=0.011$, Greenhouse-Geisser correction, $\varepsilon=0.90 ; \mathrm{c} P=0.009$, Greenhouse-Geisser correction, $\varepsilon=0.89$; $\mathrm{d}$ $\mathrm{P}=0.34$; e $\mathrm{P}=0.023$, Greenhouse-Geisser correction, $\varepsilon=0.90$. a-c Significant difference between male and female patients $(\mathrm{P}<0.01)$; no interaction between sex and across the three timepoints $(\mathrm{a} \mathrm{P}=0.20, \mathrm{~b} \mathrm{P}=0.91$, c $\mathrm{P}=0.71)$. Significant difference across the three timepoints $(\mathrm{a}-\mathrm{c} \mathrm{P}<0.01)$; for post-hoc testing, Wilcoxon singed-rank test with Bonferroni correction. $d$, e No significant difference between male and female patients ( $\mathrm{P}=0.15$, e $\mathrm{P}=0.45)$; no interaction between sex and changes across the three timepoints $(\mathrm{d} P=0.59$, e $\mathrm{P}=0.74)$. No significant difference across the three timepoints (d $\mathrm{P}=0.81$, e $\mathrm{P}=0.08)$.

This study had several limitations. Firstly, the sample size was small, particularly for comparing surgical outcomes between males and females. In addition, female sex hormones influence the strength of muscles and ligaments acting against DS, the laxity of the paraspinal ligament, and the degree of degenerative changes in the intervertebral disc. Thus, the timing of menopause should 
also be considered in females [13-15]. Secondly, the follow-up period was insufficient to evaluate the post-decompressive course following the termination of slip progression.

Table 4: Relationship between sex and reoperation.

\begin{tabular}{|c|c|c|}
\hline & Without reoperation & Reoperation \\
\hline Male (n) & 25 & 1 \\
\hline Female (n) & 58 & 5 \\
\hline
\end{tabular}

Chi-square test for independence, $\mathrm{P}=0.43$

Whether the level of decompressed DS returns to its natural course, goes on a different track from the natural course, or attains a restabilized stage earlier than the natural course remains to be determined. Thirdly, the decompression level was restricted to the L4-L5. Hence, our results on the effectiveness and limitations of MID alone for treating LSS with DS may not be generalizable to other levels of DS. This problem is also related to the influence of the decompression level and spinal alignment on surgical outcomes. Fourthly, the accuracy of the flexion/extension radiographic evaluation may depend on the pain status and cooperation of the patient; this leads to low reliability due to measurement errors and low repeatability [26]. However, these limitations do not detract from the main results of this study. The present findings unveiled the effectiveness and limitations of the unique postoperative course provided by MID alone, for LSS with DS in the context of the distinctive characteristics of DS.

\section{Conclusion}

This study revealed that MID alone for LSS with DS provided a unique postoperative course. Post-decompressive slip progression is inevitable, although it can stop one year after surgery and be followed by restabilization. The slip progression does not indicate increased mobility or secondary instability, or necessarily aggravate clinical symptoms. Interestingly, slipping in the neutral position can reduce during the second year after decompression, leading to the same degree as the preoperative condition. MID for LSS with DS can continually improve clinical symptoms even 1-2 years after surgery in both males and females. However, the time required to achieve post-decompressive restabilization, especially the approximate year that elapses before slip progression stops, is also a critical period linked to a risk of deterioration of clinical symptoms and requirement of salvage surgeries. This unique postoperative course provided by MID should be well conveyed to patients and well understood by surgeons.

\section{Acknowledgement}

We are grateful to our two late mentors for supporting this research: Dr. Baba who developed microscopic decompression called SCD for lumbar spinal stenosis and Dr. Sumida who polished SCD and made helpful suggestions for this study.

\section{References}

1. Kirdaldy-Willis WH, Farfan HF (1982) Instability of the lumbar spine. Clin Orthop Relat Res 165: 110-123.
2. Matsunaga S, Ijiri K, Hayashi K (2000) Nonsurgical managed patients with degenerative spondylolisthesis: a 10- to 18-year follow-up study. J Neurosurg 93: 194-198.

3. Enyo Y, Yoshimura N, Yamada H, Hashizume H, Yashida M (2015) Radiographic natural course of lumbar degenerative spondylolisthesis and its risk factor related to the progression and onset in a 15-year community-based cohort study: the Miyama study. J Orthop Sci 20: 978984.

4. Oertel MF, Ryang Y, Korinth MC (2006) Long-term results of microsurgical treatment of lumbar spinal stenosis by unilateral laminectomy for bilateral decompression. Neurosurg 59: 1264-1270.

5. Lee YS, Choi JC, Oh SH (2015) Semi-circumferential decompression: microsurgical total en-bloc ligamentum flavectomy to treat lumbar spinal stenosis with grade I degenerative spondylolisthesis. Clin Orthop Surg 7: 470-475.

6. Inose H, Kato T, Yuasa M (2018) Comparison of decompression, decompression plus fusion, and decompression plus stabilization for degenerative spondylolisthesis: a prospective, randomized study. Clin Spine Surg 31: E347-E352.

7. Minamide A, Simpson AK, Okada M (2019) Micro endoscopic decompression for lumbar spinal stenosis with degenerative spondylolisthesis: the influence of spondylolisthesis stage (disc height and static and dynamic translation) on clinical outcomes. Clin Spine Surg 32: E20-E26.

8. Miyauchi A, Saka S, Terayama H (2021) Efficacy and limitations of a unique postoperative course of less invasive decompression for lumbar spinal stenosis with degenerative spondylolisthesis (in Japanese). J Spine Res 12: 1152-1160.

9. Koenig S, Jauregui JJ, Shasti M (2019) Decompression versus fusion for grade I degenerative spondylolisthesis: a meta-analysis. Global Spine J 9: 155-161.

10. Pisano AJ, Butler JS, Sebastian A (2019) Does surgically managed grade I degenerative lumbar spondylolisthesis require fusion? Clin Spine Surg 32: 133-136.

11. Ilyas H, Udo-Inyang I, Savage J (2019) Lumbar spinal stenosis and degenerative spondylolisthesis: a review of the SPORT literature. Clin Spine Surg 32: 272-278.

12. Lang Z, Li JS, Yang F (2019) Reoperation of decompression alone or decompression plus fusion surgeries for degenerative lumbar disease: a systematic review. Eur Spine J 28: 1371-1385.

13. WangYX, KaplarZ, Deng M (2017) Lumbar degenerative spondylolisthesis epidemiology: a systematic review with a focus on gender-specific and age-specific prevalence. J Orhop Translat 11: 39-52.

14. Imada K, Matsui H, Tsuji H (1995) Oophorectomy predisposes to degenerative spondylolisthesis. J Bone Joint Surg 77: 126-130.

15. Taaffe DR, Sipila S, Cheng S (2005) The effect of hormone replacement therapy and/or exercise on skeletal muscle attenuation in postmenopausal women: A yearlong intervention. Clin Physiol Funct Imaging 25: 297-304.

16. Wang YX, Griffith JF, Ma HT (2011) Relationship between gender, bone mineral density, and disc degeneration in the lumbar spine: a study in elderly subjects using an eight-level MRI-based disc degeneration grading system. Ostoeporos Int 22: 91-96.

17. Miyauchi A, Sumida T, Kaneko M 92017) Morphology and clinical importance of epidural membrane and periradicular fibrous tissue in lumbar spinal stenosis. Eur Spine J 26: 382-388.

18. Fujii K, Kawamura N, Ikegami M (2015) Radiological improvements in global sagittal alignment after lumbar decompression without fusion. Spine 40: 703-709. 
19. Madkouri R, Brauge D, Buthion AV (2018) Improvement in sagittal balance after decompression surgery without fusion in patients with degenerative lumbar stenosis: clinical and radiographic results at 1 year. World Neurosurg 114: E417-E424.

20. Yagi M, Fujita N, Okada E (2018) Comparisons of direct costs, outcomes, and cost-utility of decompression surgery with fusion versus decompression alone for degenerative lumbar spondylolisthesis. J Orthop Sci 23: 653-657.

21. Försth P, Olafsson G, Carisson T (2016) A randomized, controlled trial of fusion surgery for lumbar spinal stenosis. N Engl J Med 374: 1413-1423.

22. Miyagi M, Ikeda O, Ohtori S (2013) Additional decompression at adjacent segments leads to adjacent segment degeneration after PLIF. Eur Spine J 22: 1877-1883.
23. Liu H, Wu W, Li Y (2013) Prospective effect of preserving the posterior complex on the development of adjacent segment degeneration after lumbar fusion. J Neurosurg Spine 19: 201-206.

24. Nakashima H, Kawakami N, Tsuji T (2015) Adjacent segment disease after posterior lumbar interbody fusion: based on cases with a minimum of 10 years of follow-up. Spine (Phila Pa 1976) 40: E831-E841.

25. Choi WS, Oh CH, Ji GY (2014) Spinal canal morphology and clinical outcomes of microsurgical bilateral decompression via a unilateral approach for lumbar spinal canal stenosis. Eur Spine J 23: 991-998.

26. Shen J, Xu Shen, Xu Shenxi (2018) Fusion or not for degenerative lumbar spinal stenosis: a meta-analysis and systematic review. Pain Physician 21: 1-7.

For possible submissions Click below: 